Association for Information Systems AIS Electronic Library (AISeL)

PACIS 2006 Proceedings

Pacific Asia Conference on Information Systems

(PACIS)

2006

\title{
Human Decision-Making Behavior and Modeling Effects
}

Choong Nyoung Kim

University of Seoul, cnkim27@uos.ac.kr

Kyung Hoon Yang

University of Seoul, Yang.kyun@uwlax.edu

Jaekyung Kim

University of Seoul, jkim6@unl.edu

Follow this and additional works at: http://aisel.aisnet.org/pacis2006

\section{Recommended Citation}

Kim, Choong Nyoung; Yang, Kyung Hoon; and Kim, Jaekyung, "Human Decision-Making Behavior and Modeling Effects" (2006). PACIS 2006 Proceedings. 105.

http://aisel.aisnet.org/pacis2006/105

This material is brought to you by the Pacific Asia Conference on Information Systems (PACIS) at AIS Electronic Library (AISeL). It has been accepted for inclusion in PACIS 2006 Proceedings by an authorized administrator of AIS Electronic Library (AISeL). For more information, please contact elibrary@aisnet.org. 


\title{
Human Decision-Making Behavior and Modeling Effects
}

\author{
Choong Nyoung Kim \\ Department of Management, \\ University of Seoul, Seoul, \\ Korea \\ cnkim27@uos.ac.kr
}

\author{
Kyung Hoon Yang \\ Department of Information \\ Systems, University of \\ Wisconsin-La Crosse, La \\ Crosse, WI, USA \\ Yang.kyun@uwlax.edu
}

\author{
Jaekyung Kim \\ Department of \\ Management, University of \\ Nebraska-Lincoln, Lincoln, \\ NE, USA \\ jkim6@unl.edu
}

\begin{abstract}
Previous research indicates that the human decision-making process is somewhat nonlinear and that nonlinear models would be more suitable than linear models for developing advanced decision-making models. In our study, we tested this generally held hypothesis by applying linear and nonlinear models to expert's decision-making behavior and measuring the predictive accuracy (predictive validity) and valid nonlinearity. As a result, we found that nonlinearity in the decision-making process is positively related to the predictive validity of the decision. Secondly, in modeling the human decision-making process, we found that valid nonlinearity is positively related to the predictive validity of nonlinear models. Thirdly, we found that the more nonlinearity is inherent in the decision-making process, the more nonlinear models are effective. Therefore, we suggest that a preliminary analysis of the characteristics of an expert's decision-making is needed when knowledge-based models such as expert systems are being developed. We also verify that the lens model is effective in evaluating the predictive validity of human judgment and in analyzing the validity and nonlinearity of the human decision-making process.
\end{abstract}

Keywords: Valid Nonlinearity, Predictive Validity, Expert's Decision-Making Behavior, Lens Model Analysis

\section{Introduction}

A great number of studies on human decision-making and judgment has been made in the field of social science, and a variety of methodologies and experiments have been researched (Cooksey 1996). Understanding the human decision-making process and the modeling of the decision-making process are one of the goals of this discipline (Kim and McLeod 1999; Stewart 1988). Studies on decision-making can be classified into two categories: the study of decision modeling and the study of decision process tracing (Levi 1989; Svenson 1979).

Decision modeling studies the human decision-making mechanism and tries to build models which predict human decisions. This field has been researched under the name of expert system in management discipline, and the findings are abundant (Braun and Chandler 1987; Chung and Silver 1992; Fisher and McKusick 1989; Messier and Hansen 1988; Tam and Kiang 1992). Some examples are the development of new algorithms for building decision-making models or the development of methodologies for a knowledge 
base. These technologies could currently be popularly used in the real world. In the past, studies were focused on modeling which resembled the expert's decision and judgment. However, recent studies have rigorously investigated the modeling of rules and associations using enormous amounts of real data. The application of these results has been expanded to a variety of areas, such as finance (e.g., bankruptcy prediction and stock price index prediction), marketing, account auditing, credit rating, and venture investment decision-making (Zacharakis and Meyer 1998; Velido et al. 1999; Wong et al. 2000).

Decision process tracing, the other paradigm of studies for human decision-making, focuses on the process of judgment and decision-making. This research stream has introduced various methods for the analysis of the decision-making process (Einhorn et al. 1979), such as probability scoring rules, which is used to measure the predictive validity of human judgment. Mean probability scores (MPS) is especially viewed as an efficient tool for measuring the level of uncertainty (Levi 1989; Yates 1982). Einhorn (1970; 1972) suggested Log Transformation to classify the type of human decision strategies. The lens model, proposed by Brunswick (1952) and developed by Tucker (1964), divides decisionmaking behavior into linearity and nonlinearity. It also provides the tools to measure the predictive validity of the linear parts and nonlinear parts of decision-making behavior. The effectiveness of the lens model has been verified by various empirical studies (Cooksey 1996; Levi 1989; Stewart 1988; Zimmer 1980).

Decision process tracing and decision modeling, however, are not independent because the research on both has a common goal: to improve decision quality by analyzing and understanding human decision-making behaviors. Therefore, a combination of these two areas of research presents several meaningful issues. First, a decision process tracing method such as MPS can be used in evaluating the predictive validity of decision-making models. Second, an extensive analysis of the decision process helps to develop an advanced decision-making model. For example, the lens model analysis (Tucker 1964) makes it possible to classify the contributing and non-contributing portion of an expert's predictive validity, and it helps develop a better decision-making model. Third, this combined approach may explain the reasons why statistical linear models and nonlinear models show contradictory results for the same problem. Even though many researchers (Levi 1989) insist that nonlinear models, such as the neural network model, show better performance in some studies, still, much research show that statistical linear models are better in many fields. When we take into account the fact that the performance of the model depends on the input data as well as the model itself, it would be reasonable to include characteristics of the input data, in addition to the features of decision-making behaviors when evaluating the model's predictive validity. This inclusion would be more helpful in explaining the contradictory results between previous behavioral accounting studies, which assert the superiority of the statistical linear model, and recent studies, which assert the superiority of the nonlinear model. And, this contradiction might have occurred not because of the model, but because of input data. If the predictive validity of the model is affected by both the level of the linearity/nonlinearity of the decision problem and by the validity of the input data, they should be taken into account in the selection of model development techniques.

For the above issues, we analyze the decision-making, study model building and evaluation, and investigate the relationship between the characteristics of decision- 
making behavior (nonlinearity and its validity) and predictive validity of the models.

\section{Literature Review}

\subsection{Decision Process Tracing}

The analytical framework needed to understand the human decision-making process was borrowed from studies on human judgment in the cognitive psychology discipline (Zacharakis and Meyer 1998). Study of the decision-making process is a major branch of decision-making studies, and finding the key factors affecting the decision-making process was the core research topic of previous studies. As a consequence of this research, types and characteristics of decision-making behavior and measuring methods and /or models have been developed (Einhorn et al. 1979; Olshavsky 1979). For instance, mean probability scores (MPS) was considered a useful method to measure predictive accuracy (Levi 1989). MPS is a function of squares of the deviation score between predicted values and actual outcome values. Formula of MPS is shown in Figure 1.

$$
M P S=\frac{\sum(\text { outcome }- \text { prediction })^{2}}{N}
$$

where

Outcome: actual result with values 0 or 1

Prediction: probabilistic prediction with values between 0 and 1

Figure 1: Mean Probability Score

MPS, which is an error measurement method, is widely used for assessing human's predictive validity along with the hit ratio (Levi 1989). The value of MPS is between 0 and 1 , and 'MPS $=0$ ' means that all predictions are accurate, while 'MPS $=1$ ' means all predictions are inaccurate. For example, if a certified public accountant (CPA) predicted the possibility of bankruptcy for two banks A and B with the possibility 0.7 and 0.6 respectively and they actually bankrupted later, the hit ratio is $100 \%$ and MPS is 0.125 $\left(\left[(1-0.7)^{2}+(1-0.6)^{2}\right] / 2\right)$.

Einhorn (1970) and Einhorn et al. (1979) assumed that human decision-making behavior is nonlinear, rather than linear. Furthermore, they classified human decision-making behavior into the conjunctive type and the disjunctive type, providing classification methods. Einhorn's classification has been verified by several researchers (Chung and Silver 1992).

First proposed by Brunswick (1952), the lens model has been developed by others (Hammond et al. 1964; Tucker 1964) to investigate the use of nonlinearity in human decision-making behavior. Many behavioral accounting researchers have discussed the use of the lens model with regard to the examination of a judgment situation in which a human makes decisions (Libby 1981). Detailed description of the lens model appears in Kim and McLeod (1999)

\subsection{Research on the Decision-Making Model}


Since the 1960s, research on decision-making has been explored in accounting and management sciences, as well as in many other fields. One of the main concerns was how to mimic the human decision-making and whether the model predicts better than human experts do. Key modeling methods are linear, such as linear regression analysis and discriminant analysis. Most experimental studies found that linear models may predict the actual outcome more accurately than human decision does (Dawes and Corrigan 1974; Levi 1989), although there is research that shows otherwise (Libby 1976; Schepanski 1983). Previous researchers in this field state that this kind of positive results happens due to the simple linearity of the model, which reduced the inconsistency of human decision behavior.

Studies on the decision-making model continued with the expert system and artificial intelligence research in the fields of management since the late 1970s. Several modeling methodologies and algorithms were researched to extract the expert's knowledge and decision-making behavior. One of these methodologies is the inductive learning approach, which treats input data as nonlinear. Quinlan's (1979) ID3 (also evolved to C5 later) is the most widely used algorithm in inductive learning approaches. ID3 represents the human decision process as a tree structured model and shows a very prominent prediction accuracy compared to traditional statistical approaches. ID3 shows a higher prediction accuracy, especially when sample data are stable with less noise (Kim and McLeod 1999). In its earlier stage of development, ID3 could only treat discrete data and make a binary classification; however, as it has evolved, it can also handle continuous data, making possible a more sophisticated classification. The other approach is a neural network, which is broadly applied in modeling of management decision-making. Neural network models are widely used in a variety of applications because they are free from statistical assumption, making it easy to find nonlinear relationships among input and output variables. Furthermore, it shows better performance in dealing with noisy sample data (Wong et al. 2000).

\subsection{Comparative Studies for the Linear Model and Nonlinear Model}

The decision-making model is mainly applied to classification and/or prediction problems. Most classification researchers have used a hit ratio for the performance evaluation criterion. They have also used statistical models, such as regression analysis, discriminant analysis, and logistic analysis, which are based on linear relationships among variables (Chung and Silver 1992). These statistical models have been mainly used to analyze and model an expert's decision-making behavior in behavioral sciences, such as psychology and behavioral accounting, where they have proved their prowess (Belkaoui 1989; Casey 1983; Dawes and Corrigan 1974). Due to the superiority of linear models, the evaluation of nonlinear models such as the neural network, tree structure algorithm, and genetic algorithm was measured by comparing their results with those of statistical models (Chung and Silver 1992).

However, Chung and Silver (1992) argued that the comparison of both sets of research is only based on input data without considering the type of tasks or decision-making behavior to which the methods are applied. Previous research on expert systems also committed these kinds of mistakes without considering circumstantial factors such as the characteristics of input data and expert's behavior in the research model design. Characteristics of data or those of algorithm in models may also distort the model 
performance. For example, the linearity and nonlinearity of input data can distort the performance of a model when the linear model analyzes the nonlinearity of input data or the nonlinear model analyzes the linearity of input data.

Therefore, a comparison study that considers both factors is needed. To overcome this limitation, an analysis on environmental factors, such as the characteristics of input data and the participating expert's behavior, should come first, and the result of the preliminary analysis should be used in the performance evaluation of models. There is a second issue. The hit ratio was popularly used as a performance index in the expert systems because they had been mainly used for classification. In this case, much information can be lost since the decision is always either ' 0 ' or ' 1 '. Therefore, the hit ratio may not be appropriate as a performance measurement because it does not reflect the level of uncertainty and/or competence of the expert's knowledge, experience, or judgment. To overcome this limitation, an additional performance index is required to measure the predictive validity more accurately.

Consequently, it is believed that when evaluating model performance, the model should include environmental factors such as the characteristics of input data and participating expert's behavior, as well as the uncertainty and competence of the expert's knowledge, experience, or judgment.

\subsection{Research Objectives}

It seems that a combination of research on decision-making modeling and on decision process tracing would be valuable not only theoretically but also practically as Svenson (1979) has insisted. But, despite his intention to that effect, there has been a lack of effort put into this kind of research. In this study, we analyze the findings and methods of both pieces of research and combine them to overcome the weakness of each. We expect to find a relationship between characteristics of decision-making behavior and modeling methods by comparing the performance of modeling methods based upon an analysis of the characteristics of decision-making behavior. This study has three objectives. First, we analyze the experts' decision-making strategies in terms of linearity, nonlinearity, and validity in order to find the specific behavioral characteristics contributing to predictive validity. Second, we adopt MPS, in addition to hit ratio, to measure the accuracy of predictive models which were built based upon a linear or nonlinear algorithm. Third, we look for the relationships between prediction models and decision-making behaviors. Based upon the above results, we seek to find which model is more valid for prediction when nonlinearity is inherent in the input data and/or decision-making behavior. This approach may reveal that the conflicting results of previous comparative studies between statistical linear models and nonlinear prediction models were caused by the nonlinear characteristics of decision-making behavior rather than the nonlinear properties of the input data.

\subsection{Research Model and Hypotheses}

Three hypotheses are built based upon previous research.

Hypothesis 1: Validity (Accuracy) of decision-making is positively related to the valid nonlinearity of decision-making. 
This hypothesis is based upon the presumption: human decision-making is basically nonlinear, and the validity of decision-making is decided by valid nonlinearity. Validity means the accuracy of decision-making, and valid nonlinearity is defined as the nonlinearity portion that affects the accuracy of decision-making. In other words, the higher the accuracy of decision-making, the higher valid nonlinearity. Therefore, we assume the nonlinearity of experts' decision-making is higher than that of non experts.

Hypothesis 2: Valid nonlinearity of decision-making is positively related to the prediction accuracy of a nonlinear model, but not positively related to the prediction accuracy of a linear model.

This hypothesis is based upon the presumption: valid nonlinearity of decisionmaking is explained better by a nonlinear model than by a linear one. It is related to the selection of the modeling method. We assume that a nonlinear model leads to better predictive validity than a linear model if there is valid nonlinearity in input data or decision-making behavior. We also assume that the reason the superiority of a linear model has been reported in previous studies (Dawes and Corrigan 1974; Levi 1989) is that there is no valid nonlinearity in the input data or in decision-making behavior.

Hypothesis 3: The modeling effect of a nonlinear model increases than that of a linear model as the validity of decision-making increases.

The modeling effect is defined as the improvement of the predictive validity by modeling the human decision-making behavior. It is expressed as the residual value of subtracting the predictive validity of human judgment from the predictive validity of the model. Therefore, in this study, we explore the relationship between the model's predictive validity and decision-maker's behavioral characteristics by analyzing the decisionmaking behavior, building decision models, and evaluating them concurrently. Figure 2 depicts our research model and hypotheses.

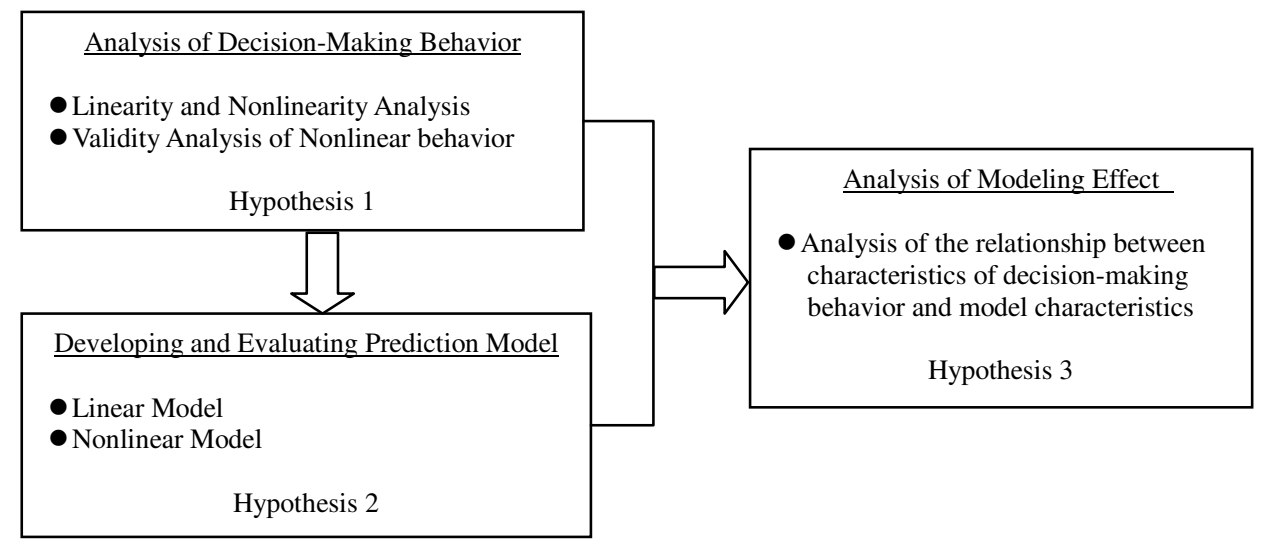

Figure 2. Research Model

\section{Research Method}

\subsection{Task: Bankruptcy Prediction}


We choose bankruptcy prediction as the experimental task to test our research hypotheses. Bankruptcy prediction is one of the most frequently studied human decision-making tasks since Altman's research (1968). As a result, there are a great deal of previous studies we can refer to, and to which we can compare our study. Bankruptcy prediction is also directly related to many applications, such as credit rating, bank loan, and venture investment decisions (Zacharakis and Meyer 1998; Tam and Kiang 1992).

\subsection{Data and Participants}

Our data were obtained from thirty bankrupted and thirty non-bankrupted companies in the U.S. in 1985. To maintain the consistency of data quality, we extracted sample companies from the same industry (manufacturing) with a similar size of about $\$ 50$ million in average assets. We used financial data for two years prior (1983) to bankruptcy for each company. We kept the names of the companies anonymous. The most frequently used ten financial ratios in the previous studies (Kim and McLeod 1999; Harris 1989) were considered; 1) Net Income/Total Assets (Profitability), 2) Current Assets/Sales (Activity), 3) Current Assets/Current Liability (Liquidity Ratio), 4) Current Assets/Total Assets (Asset Balance), 5) Cash/Total Assets (Cash Position), 6) Total Debt/Total Assets (Financial Leverage), 7) [Current Assets - Current Liability]/Total Assets (Relative Working Capital), 8) Sales/Total Assets (Sales-Generating Ability of Assets), 9) Retained Earnings/Total Assets (Cumulative Profitability), 10) [Current Asset/Current Liability]/Sales (Working Capital Turnover)

Participants are selected from two groups: one group consists of 16 experts who work as certified public accountants (CPA) currently or financial CEOs of big companies who have experience as a CPA; the other consists of 24 graduate students majoring in Finance and Accounting. To increase reliability, we adopted the test-retest approach. First, participants were asked to predict the bankruptcy/no-bankruptcy of 70 cases, in which ten cases were asked twice (total 60 different cases). The participants whose prediction of ten duplicated cases was lower than $80 \%$ of consistency were eliminated. Eight students were eliminated, and 32 participants were selected. We expected that the prediction accuracy and the decision-making behaviors of the two groups would be different.

\subsection{Experiment Procedure}

The experiment in this study consists of three steps. In the first step, each participant predicts the bankruptcy possibility of 60 sample companies. Each does two types of prediction: binary decision and probability prediction. In a binary decision, each company is labeled as either ' 0 ' (bankruptcy) or ' 1 ' (no-bankruptcy). In probability prediction, each company is labeled by a ten-level, quasi-continuous scale based on the participant's confidence in the decision. If a participant predicts bankruptcy for a company, he/she may choose from ' 0.0 ' to ' 0.4 ,' where ' 0.0 ' implies the highest confidence and ' 0.4 ' the lowest confidence of bankruptcy. If a participant predicts nobankruptcy, he/she chooses a value from '0.6' to '1.0,' where '1.0' means the highest confidence and ' 0.6 ' the lowest confidence of no-bankruptcy. The reason that we use both the binary decision and probability prediction is that certain types of algorithms cannot be fairly evaluated if the object variable is considered as any one type of either discrete or continuous.

In the second step, the prediction performance of each participant is evaluated by the hit 
ratio and MPS. The portion of nonlinearity in the decision-making and the validity of nonlinearity is also examined by the lens model's C-Index and $\mathrm{R}_{\mathrm{a}}$. The lens model consists of two types of linear models. One is the linear regression model of participants' bankruptcy prediction and the independent variables of ten financial ratios, and the other is the regression model of the actual result of bankruptcy and the independent variables. Quasi-continuous value of participant's prediction confidences were used as a dependent variable in the regression model.

In the third step, prediction models are developed based on each participant's prediction. Statistical linear regression is adopted for the linear model, and Quinlan' (1979) C4.5 and the back-propagation paradigm of neural network is adopted for the nonlinear model. It is known that the number of hidden layer is positively related to the overfitting of training, and it is recommended to use fewer numbers of hidden layers than the number of input node (Patuwo et al. 1993; Wong et al. 2000) in building the neural network model. Though there is no rule for the exact number of hidden layers, many previous studies used one hidden layer (Kim and McLeod 1999; Wong et al. 2000). In our research, the neural network model consists of ten input nodes, five hidden nodes in one hidden layer, and one output node. The sigmoid function was used for the transfer function, and the delta rule was used for the learning algorithm. We repeatedly used this network model 32 times for each participant. The participants' prediction confidence level was used as the objective variable in the training samples, while actual bankruptcy was used in the testing samples. This makes it possible to divide training samples and testing samples and to use both hit ratio and MPS for the measurement of predictive validity of linear or nonlinear models.

\section{Analysis and Result}

\subsection{Analysis of Decision-Making}

The prediction accuracies of 32 participants were measured by hit ratio, MPS, and $\mathrm{R}_{\mathrm{a}}$ of the lens model. Valid nonlinearity of participants' decision-making behavior is measured with C-Index of the lens model. Hit ratios of most of the participants are around $70 \%$, and the highest is $83 \%$ (participant \# 3). Most of MPS are around 0.2. Values of $R_{a}$ are greater than the values of previous studies (Kim and McLeod 1999). We assume the reason for this is because predictive variable is not discretely, but quasi-continuously measured with ten-level prediction confidence. C-Index which shows the validity of the nonlinearity of 12 participant's decisions was valid, with a significant level of $\alpha=.05$, and 5 participants were valid, with a significance level of $\alpha=0.1$. The results of the prediction accuracy of 32 participants are summarized in Table 1 . 


\begin{tabular}{|c|c|c|c|c|c|}
\hline Participant & $\mathbf{R a}$ & Hit Ratio & MPS & C-Index & $\begin{array}{c}\text { C-Index } \\
(t \text {-stat. })\end{array}$ \\
\hline 1 & 0.552 & $78 \%$ & 0.1767 & 0.245 & *1.92451 \\
\hline 2 & 0.630 & $80 \%$ & 0.1548 & 0.528 & 4.73494 \\
\hline 3 & 0.657 & $83 \%$ & 0.1430 & 0.534 & 4.81005 \\
\hline 4 & 0.573 & $77 \%$ & 0.1757 & 0.287 & 2.28171 \\
\hline 5 & 0.579 & $75 \%$ & 0.1708 & 0.236 & 1.84956 \\
\hline 6 & 0.511 & $73 \%$ & 0.2057 & 0.244 & 1.91616 \\
\hline 7 & 0.546 & $78 \%$ & 0.1758 & 0.186 & *1.44169 \\
\hline 8 & 0.447 & $73 \%$ & 0.2105 & 0.104 & 0.79635 \\
\hline 9 & 0.584 & $80 \%$ & 0.1655 & 0.310 & 2.48322 \\
\hline 10 & 0.426 & $68 \%$ & 0.2180 & 0.026 & 0.19807 \\
\hline 11 & 0.489 & $70 \%$ & 0.2115 & 0.289 & **2.299061 \\
\hline 12 & 0.517 & $75 \%$ & 0.1963 & 0.15 & 1.155439 \\
\hline 13 & 0.609 & $77 \%$ & 0.1708 & 0.359 & ** 2.929339 \\
\hline 14 & 0.496 & $75 \%$ & 0.1962 & 0.203 & * 1.578876 \\
\hline 15 & 0.399 & $70 \%$ & 0.232 & -0.051 & -0.38891 \\
\hline 16 & 0.517 & $68 \%$ & 0.2048 & 0.281 & ** 2.229879 \\
\hline 17 & 0.558 & $72 \%$ & 0.1802 & 0.209 & *1.627642 \\
\hline 18 & 0.496 & $73 \%$ & 0.1933 & 0.048 & 0.365979 \\
\hline 19 & 0.488 & $72 \%$ & 0.1932 & 0.157 & 1.210691 \\
\hline 20 & 0.363 & $65 \%$ & 0.2648 & 0.154 & 1.186989 \\
\hline 21 & 0.532 & $70 \%$ & 0.1948 & 0.259 & 2.04217 \\
\hline 22 & 0.569 & $73 \%$ & 0.1732 & 0.201 & *1.562662 \\
\hline 23 & 0.483 & $70 \%$ & 0.2083 & 0.253 & **1.991584 \\
\hline 24 & 0.394 & $70 \%$ & 0.2553 & 0.057 & 0.434806 \\
\hline 25 & 0.307 & $63 \%$ & 0.261 & 0.004 & 0.030463 \\
\hline 26 & 0.342 & $63 \%$ & 0.2277 & -0.101 & -0.77315 \\
\hline 27 & 0.425 & $68 \%$ & 0.2473 & 0.051 & 0.388911 \\
\hline 28 & 0.444 & $70 \%$ & 0.2072 & -0.097 & -0.74223 \\
\hline 29 & 0.338 & $67 \%$ & 0.2643 & 0.202 & *1.570767 \\
\hline 30 & 0.363 & $68 \%$ & 0.2587 & 0.157 & 1.210691 \\
\hline 31 & 0.249 & $62 \%$ & 0.2768 & -0.111 & -0.85061 \\
\hline 32 & 0.348 & $72 \%$ & 0.2475 & -0.001 & -0.00762 \\
\hline
\end{tabular}

Table 1. Prediction Accuracy Analysis of Participant

Table 2 shows the correlation among $\mathrm{R}_{\mathrm{a}}$, hit ratio, MPS, and C-Index. The correlation coefficient between $R_{a}$ and hit ratio is relatively high at 0.8701 , and this means that $R_{a}$, which is used to measure predictive validity in the lens model, might be an appropriate measurement for prediction accuracy. Correlation coefficients between $\mathrm{R}_{\mathrm{a}}$ and MPS and between hit ratio and MPS both are -0.8598 , and this high correlation may come from the fact that MPS also measures prediction accuracy. MPS shows the negative relationships with other indexes because it uses the prediction error which caused the negative sign. Correlation coefficients between C-Index (measuring valid nonlinearity) and prediction accuracy measurements are high - the $\mathrm{R}_{\mathrm{a}}$ is 0.8026 , the hit ratio is 0.7129 , and the MPS is -0.6889 - and statistically significant at $\alpha=.01$ level. Therefore, we can conclude that valid 
nonlinearity is highly correlated with the validity of decision-making, and hypothesis 1 is accepted.

\begin{tabular}{|c|c|c|c|}
\hline & Hit Ratio & MPS & C-Index $\quad$ (p-value) \\
\hline $\mathbf{R}_{\mathbf{a}}$ & 0.8701 & -0.8598 & $0.8026(\mathrm{p}<0.0001)$ \\
\hline Hit Ratio & - & -0.8598 & $0.7129(\mathrm{p}<0.0001)$ \\
\hline MPS & - & - & $-0.6889(\mathrm{p}<0.0001)$ \\
\hline
\end{tabular}

Tabel 2. Correlation between C-Index and Prediction Accuracy

These results support the findings of previous studies (Levi 1989): valid nonlinearity is an important factor contributing to predictive validity. These results also mean that valid nonlinearity can be used to measure predictive validity. To examine the value of C-Index as an evaluation index of prediction accuracy, we classified the samples as valid group (with 0.2 or higher of C-Index value) and invalid group (less than 0.2 ) and compared the two groups' prediction accuracy. Two groups have statistically different ( $p$-value $<0.001$ ) prediction accuracies in terms of $\mathrm{R}_{\mathrm{a}}$, hit ratio, and MPS, and this result shows that valid nonlinearity-based classification is consistent with the classification based on predictive accuracy. Table 3 shows the results in detail.

\begin{tabular}{|c|c|c|c|c|}
\hline Group & C-Index & $\mathbf{R}_{\mathbf{a}}$ & Hit Raio & MPS \\
\hline Valid Group (n=16, C-Index > 2.0) & 0.290 & 0.542 & $74.25 \%$ & 0.187 \\
\hline Invalid Group (n=16, C-Index <2.0) & 0.046 & 0.409 & $69.38 \%$ & 0.229 \\
\hline$t$-stat. (p-value) & $\begin{array}{c}6.781 \\
(\mathrm{p}<0.001)\end{array}$ & $\begin{array}{c}4.865 \\
(\mathrm{p}<0.001)\end{array}$ & $\begin{array}{c}2.998 \\
(\mathrm{p}<0.001)\end{array}$ & $\begin{array}{c}3.980 \\
(\mathrm{p}<0.001)\end{array}$ \\
\hline
\end{tabular}

Table 3. Prediction Accuracy of Participant (Group Average)

\subsection{Model Construction and Evaluation}

Decision-making models were developed using a linear regression model, tree structure model using C4.5, and neural network model using back propagation and applied to 32 participants. Hit ratio and MPS are used as evaluation criteria. In the linear regression and neural network model, we used the value 0.4 as a threshold to evaluate hit ratio because prediction values are continuous values in these two models. This threshold is also used for the tree structure model for consistency purposes. It is reasonable that the value 0.4 is counted as a threshold, because we classified the non-bankrupt company with a prediction confidence of greater than 0.6 and the bankrupt company with less than 0.4 in the training sample. 0 - 0.4 means bankruptcy and $0.6-1.0$ means non-bankruptcy. The tree structure model may be more unfairly evaluated than the linear regression model or neural network model when measuring MPS, because the object variable of the linear regression model and neural network model is continuous, while that of the tree structure model is discrete. However, in this study, we calculated MPS under the assumption that the objective variable of the tree structure model is continuous because the objective variable is measured by quasi-continuous scale values $(0.0-1.0)$. 


\begin{tabular}{|c|c|c|c|c|c|c|}
\hline \multirow{2}{*}{ Group } & \multicolumn{2}{|c|}{$\begin{array}{c}\text { Linear Regression } \\
\text { Model }\end{array}$} & \multicolumn{2}{c|}{$\begin{array}{c}\text { Tree Structure } \\
\text { Model }\end{array}$} & \multicolumn{2}{c|}{$\begin{array}{c}\text { Neural Network } \\
\text { Model }\end{array}$} \\
\cline { 2 - 7 } & Hit Ratio & MPS & Hit Ratio & MPS & Hit Ratio & MPS \\
\hline Valid Group (n=16, C-Index > 2.0) & $61.14 \%$ & 0.195 & $71.46 \%$ & 0.187 & $73.23 \%$ & 0.187 \\
\hline Invalid Group (n=16, C-Index < 2.0) & $57.08 \%$ & 0.201 & $67.10 \%$ & 0.210 & $65.94 \%$ & 0.228 \\
\hline t-value & $2.206^{*}$ & -0.88 & $1.697 *$ & $-2.34 *$ & $4.883^{*}$ & $-3.95^{*}$ \\
\hline
\end{tabular}

${ }^{*} \alpha<0.05$

Table 4. Group Performance Comparison among Prediction Models

In Table 4, we classified participants into two groups of valid and invalid based on CIndex and analyzed the two groups by linear regression, tree structure, and neural network. After that, we calculated the average of prediction accuracy of the models for each group. Since the purpose of this study is not model comparison but the investigation of the effect of valid nonlinearity in modeling, we skipped the model performance comparison. The difference between groups indicates that valid nonlinearity of decision behavior is directly related to predictive validity of the model. The results of the two groups, which are classified by C-Index, show significant differences in the hit ratio and MPS between the tree structure model and the neural network model. However, the hit ratio in the linear regression model also shows a significant difference between two groups; thus, hypothesis 2 cannot be accepted. We can only infer that the valid nonlinearity has an effect on predictive validity based upon the results of nonlinear models. We may also assume that MPS is more suitable than the hit ratio because MPS is used for continuous value. If the above assumption is correct, and if we could give more weight to MPS than hit ratio, we might conjecture that no significant relationship exists between valid linearity and the predictive validity of the linear model.

\subsection{Comprehensive Comparison}

Table 5 shows the top ten models and participants based on hit ratio. Four participants and six models - three tree structure models and three neural network models - are included, but a linear regression model was not. It can be interpreted that as the expert's prediction accuracy increases, the modeling effect of linear models decreases. The highest hit ratio $(83.3 \%$ ) is achieved by the tree structure model of participant 3 , and this value is even higher than actual human judgment (participant 3 ). The predominance of model over human judge is also found for participant 1 , whose neural network models $\left(7^{\text {th }}\right)$ outperform the human prediction $\left(10^{\text {th }}\right)$. C-Indexes of participants $(1,2,3,9$, and 13$)$ whose models are ranked in the top 10 are found to be the highest values among 32 participants. 


\begin{tabular}{|l|c|c|}
\hline \multicolumn{1}{|c|}{ Participant / Model } & Hit Ratio & Rank \\
\hline Tree structure model (Participant 3) & $83.3 \%$ & 1 \\
\hline Participant 3 (C-Index: 0.534) (1 $^{\text {st }}$ ranked participant in prediction accuracy) & $83.0 \%$ & 2 \\
\hline Neural network model (Participant 9) & $80.0 \%$ & 3 \\
\hline Tree structure model (Participant 9) & $80.0 \%$ & 3 \\
\hline Participant 9 (C-Index: 0.310) $\quad$ (2 $^{\text {nd }}$ ranked participant in prediction accuracy) & $80.0 \%$ & 3 \\
\hline Participant 2 (C-Index: 0.528) $\left(3^{\text {rd }}\right.$ ranked participant in prediction accuracy) & $80.0 \%$ & 3 \\
\hline Neural network model (Participant 1) & $78.3 \%$ & 7 \\
\hline Neural network model (Participant 13) & $78.3 \%$ & 7 \\
\hline Tree structure model (Participant 2) & $78.3 \%$ & 7 \\
\hline Participant 1 (C-Index: 0.245) (4 $^{\text {th }}$ ranked participant in prediction accuracy) & $78 \%$ & 10 \\
\hline
\end{tabular}

Table 5. Top 10 Ranking of Prediction Accuracy Using Hit Ratio

Table 6 lists the top ten models and participants based on MPS. The result is similar to Table 5. Three human experts (participant 2, 3, and 9), four tree structure models, and three neural network models are included but a linear model is not. The highest prediction accuracy was achieved by the tree structure model of participant \#3, the same result shown in Table 5. The predominance of models over human experts was found in the case of participant \#3, where his/her model $\left(1^{\text {st }}\right)$ outperforms his/her judgment $\left(2^{\text {nd }}\right)$ and in the case of participant \#9, whose neural network model $\left(6^{\text {th }}\right)$ outperforms his/her judgment $\left(8^{\text {th }}\right)$. The same participants $(\# 1,2,3$, and 9) whose models are ranked top ten in Table 5 are also listed in Table 6 . The results of Table 5 and 6 show that there is valid nonlinearity contributing to prediction accuracy in the decision-making behavior and nonlinear models reflect nonlinearity of behavior better than the linear model.

Based on the results of Table 5 and 6 , we measured the correlation between the prediction accuracy of participants and that of models by hit ratio and $\mathrm{R}_{\mathrm{a}}$. We also measured the correlation between participants' prediction accuracy and modeling effect. Modeling effect is measured as the difference between the models' prediction accuracy and the participants' prediction accuracy. Hence, it shows how much the model increased the accuracy of the decision-making. Generally, it is believed that the higher the participants' prediction accuracy is, the lower the modeling effect. Therefore, analyzing the correlation between the participants' prediction accuracy and modeling effect allows us to find which modeling effect significantly decreases as the participants' prediction accuracy increases. In this analysis, MPS is not used because it has a negative relationship to other prediction measurements. Thus, it should be converted to compare with the other measurements. MPS is also very highly correlated (-0.8598 in Table 2$)$ with the hit ratio and $R_{a}$. 


\begin{tabular}{|l|c|c|}
\hline \multicolumn{1}{|c|}{ Model/ Participant } & MPS & Rank \\
\hline Tree structure model (Participant 3) & 0.1387 & 1 \\
\hline Participant 3 (C-Index: 0.534) (Top ranked participant in prediction accuracy) & 0.1430 & 2 \\
\hline Neural network model (Participant 3) & 0.1434 & 3 \\
\hline Tree structure model (Participant 2) & 0.1548 & 4 \\
\hline Participant 2 (C-Index: 0.528) (2 $^{\text {nd }}$ ranked participant in prediction accuracy) & 0.1548 & 4 \\
\hline Tree structure model (Participant 9) & 0.1583 & 6 \\
\hline Neural network model (Participant 2) & 0.1608 & 7 \\
\hline Neural network model (Participant 9) & 0.1647 & 8 \\
\hline Participant 9 (C-Index: 0.310) (3 ${ }^{\text {rd }}$ ranked participant in prediction accuracy) & 0.1655 & 9 \\
\hline Tree structure model (Participant 1) & 0.1672 & 10 \\
\hline
\end{tabular}

Table 6. Top Ten Ranking of Prediction Accuracy using MPS

Table 7 shows the results of the analysis. The neural network model is most affected by the participant's prediction accuracy (correlation coefficient of 0.8729 with hit ratio and 0.8006 with $\mathrm{R}_{\mathrm{a}}$ ). Generally, the modeling effect is negatively related to the participant's prediction accuracy. However, this negative correlation is found to be statistically significant only in the linear regression model ( -0.3925 in hit ratio and -0.3172 in $\left.R_{a}\right)$. This is consistent with the result found in Table 5 and 6, implying that modeling expert's decision-making behavior using a linear model significantly decreases the modeling effect when his predictive validity is high. We could interpret that this happened because the linear model can not reflect valid nonlinearity, which ensures experts' predictive validity. This leads us to accept our third hypothesis: that the modeling effect of the nonlinear model increases more than that of the linear model as the validity of decisionmaking increases.

\begin{tabular}{|c|c|c|c|}
\hline \multirow{2}{*}{ Model Prediction Accuracy and Modeling Effect } & \multicolumn{2}{|c|}{ Participants' Prediction Accuracy } \\
\cline { 3 - 4 } & Hit Ratio & $\mathbf{R}_{\mathbf{a}}$ \\
\hline \multirow{2}{*}{$\begin{array}{c}\text { Model Prediction } \\
\text { Accuracy }\end{array}$} & Linear Regression Model & 0.5866 & 0.5318 \\
\cline { 2 - 4 } & Tree Structure Model & 0.6005 & 0.5521 \\
\cline { 2 - 4 } & Neural Network Model & 0.8729 & 0.8006 \\
\hline \multirow{3}{*}{\begin{tabular}{c} 
Modeling Effect \\
\cline { 2 - 4 }
\end{tabular}} & Linear Regression Model & $\mathbf{- 0 . 3 9 2 5 *}$ & $-\mathbf{0 . 3 1 7 2} *$ \\
\cline { 2 - 4 } & Tree Structure Model & -0.1063 & -0.0557 \\
\cline { 2 - 4 } & Neural Network Model & -0.1105 & -0.0127 \\
\hline
\end{tabular}

Table 7. Correlation between Participants' Prediction Accuracy and Modeling Effect

\section{Conclusion}

It is believed that human decision-making behavior is quite nonlinear and that using a nonlinear model would be more effective in decision making (Levi 1989; Olshavsky 1979; Einhorn et al. 1979). In this study, to test this hypothesis, we developed models of an expert's decision using linear and nonlinear models and investigated the relationship between valid nonlinearity and predictive validity. We discovered several interesting results. First, we found a significant relationship between valid nonlinearity and predictive validity through the analysis of human decision-making behavior using hit ratio, MPS, and the lens model. Second, nonlinear models showed a higher prediction 
accuracy than linear models when nonlinearity was inherent in the participant's decision behavior. Third, we found that there is a negative relationship between the nonlinearity of human decision-making behavior and the prediction accuracy of linear models. We interpreted this to mean that the more valid nonlinearity there is, the less effective the use of linear models. These findings explain the conflicts in previous research results. The conflict is not because of the difference of the models, but because of the difference of input data characteristics, such as nonlinearity. It seems that when linear models were better than the nonlinearity model, there was significant noise in the input data and/or the linear model was developed based on the non-expert's decision behavior. Therefore, it is possible that there is less valid nonlinearity in the data, while previous research which showed the superiority of the nonlinear model to the linear model, may adopt the expert's decision behavior or less-noisy data as input data. Consequently, it would be better to select the modeling algorithm based upon the analysis of the characteristics of input data and/or consideration of human expert's decision-making behavior.

We believe the results of our research might raise research issues in the field of expert systems and decision models. First, the lens model divides prediction accuracy into linear and nonlinear components and provides different evaluation criteria. A lens model can answer the question of whether a nonlinear model can show better prediction accuracy than a linear model or even human experts' judgment.

The second issue involves knowledge management. Knowledge base is the core component of a knowledge-based model. The fact that the predictive validity of experts, which provides a knowledge base, conveys a critical role on the prediction accuracy of the model has been proven by much previous research (Levi 1989; Libby 1981; Zimmer 1980). We verified the predictive validity and valid nonlinearity of experts, using a lens model. Based upon this result, training the experts might improve their predictive validity, which would eventually improve the prediction accuracy of the decision-making model. Decision-making research, combined with expert system research, can expand to a variety of applications such as finance, medical science, and credit rating. However, a more advanced decision model development requires systematic and synthesized study, which includes empirical studies of various areas and circumstantial factors affecting a system's prediction accuracy. We hope that this research will be helpful to future research in considering more possible circumstantial factors.

\section{Reference}

Altman, E., "Financial Ratios, Discriminant Analysis and the Prediction of Corporate Bankruptcy," Journal of Finance (23:4), 1968, pp. 589-609.

Beach, L. and Mitchell, T., "A Contingency Model for the Selection of Decision Strategies," Academy of Management Review (3:3), 1978, pp. 439-449.

Belkaoui, A., "Lens Studies in Accounting," in Human Information Processing in Accounting, A. Belkaoui, (ed), Quorum Books, New York, 1989.

Billings, R. and Marcus, S., "Measures of Compensatory and Noncompensatory Models of Decision Behavior," Organizational Behavior Human performance (31:3), 1983, pp. 331-352.

Braun, H. and Chandler, J., "Predicting Stock Market Behavior through Rule Induction," Decision Sciences (18:3), 1987, pp. 415-429.

Brunswick, E., The Conceptual Framework of Psychology, University of Chicago Press, 
Chicago, 1952.

Casey, C. J., "Prior Probability Disclosure and Loan Officers' Judgment: Some Evidence of the Impact," Journal of Accounting Research (21:1), 1983, pp. 300-307.

Chung, H. M. and Silver, M. S., "Rule-Based Expert Systems and Linear Models: An Empirical Comparison of Learning-by-Examples Methods," Decision Sciences (23:3), 1992, pp. 687-707.

Cooksey, R., Judgment Analysis: Theory, Methods, and Applications, San Diego Academic Press, 1996.

Dawes, R. and Corrigan, B., "Linear Models in Decision-Making," Psychological Bulletin (81:2), 1974, pp. 95-106.

Einhorn, H., "The Use of Nonlinear, Noncompensatory Models in Decision-Making," Psychological Bulletin (77:3), 1970, pp. 221-230.

Einhorn, H., "Expert Measurement and Mechanical Combination," Organizational Behavior Human Performance (20:2), 1972, pp. 86-196.

Einhorn, H., Kleimuntz, D., and Kleimuntz, B., "Linear Regression and Process-tracing Models of Judgment," Psychological Review (86:6), 1979, pp. 465-485.

Fisher, D. H. and McKusick, K. B., "An Empirical Comparison of ID3 and BackPropagation," Technical Report CS-88-14, Dept. of Computer Science, Vanderbilt University, 1989.

Harris, C., "An Expert Decision Support Systems for Auditor Going Concern Evaluation," Ph.D. Dissertation, University of Texas at Arlington, 1989.

Hammond, K., Hursch, C., and Todd, F., Analyzing the Components of Clinical Inference, Psychological Review (71:6), 1964, pp. 438-456.

Kim, C. and McLeod, R., "Expert, Linear Models, and Nonlinear Models of Expert Decision-Making in Bankruptcy Prediction," Journal of Management Information Systems (16:1), 1999, pp. 189-207.

Levi, K., "Expert Systems Should Be More Accurate Than Human Experts," IEEE Transactions on Systems, Man, and Cybernetics (19:3), 1989, pp. 647-657.

Libby, R., "Man versus Model of Man: Some Conflicting Evidence," Organizational Behavior Human Performance (16:1), 1976, pp. 1-12.

Libby R., Accounting and Human Information Processing: Theory and Applications, Englewood Cliffs, Prentice Hall, New Jersey, 1981.

Messier, W. and Hansen, J., "Inducing Rules for Expert System Development: An Example Using Default and Bankruptcy Data," Management Science (34:12), 1988, pp. 1403-1415.

Olshavsky, R. W., "Task Complexity and Contingent Processing in Decision-Making: A Replication and Extension," Organizational Behavior Human Performance (24:3), 1979, pp. 300-316.

Patuwo, E., Hu, M. and Hung, M., "Two-Group Classification Using Neural Networks," Decision Sciences (24:4), 1993, pp. 825-845.

Quinlan, J., "Discovering Rules by Induction from Large Collection of Examples," in Expert Systems in Microelectronic Age, D. Michie (ed.), Edinburgh University Press, Edinburgh, 1979, pp. 169-201.

Schepanski, A., "Tests of Theories of Information Processing Behavior in Credit Judgment," Accounting Review (58:3), 1983, pp. 581-599.

Steawart T., "Judgment Analysis: Procedures, " in Human Judgment: the SJT View, B. 
Brehmer and R. B. Joyce (eds.), Amsterdam North-Holland Elsevier, 1988, pp. 41-74. Svenson, O., "Process Descriptions of Decision-Making," Organizational Behavior Human Performance (23:1), 1979, pp. 86-112.

Tam, K. and Kiang, M., "Managerial Applications of Neural Networks: The Case of Bank Failure Predictions," Management Science (38:7), 1992, pp. 926-947.

Tucker, L. R., "A Suggested Alternative Formulation in the Development of Hursch, Hammond, and Hursch, and by Hammond, Hursch, and Todd," Psychological Review (71:6), 1964, pp. 458-530.

Velido, A., Lisboa, P., and Vaughan, J., "Neural Networks in Business: A Survey of Applications (1992-1998)," Expert Systems with Applications (17), 1999, pp. 51-70.

Wong, B., Lai, V., and Sam, J., " A Bibliography of Neural Network Business Applications Research: 1994-1998," Computers \& Operations Research (27), 2000, pp. 1045-1076.

Yates, J. F., "External Correspondence: Decompositions of the Mean Probability Score," Organizational Behavior Human Performance (30), 1982, pp. 132-156.

Zacharakis, A., and Meyer, G., "A Lack of Insight: Do Venture Capitalists Really Understand Their Own Decision Process," Journal of Business Venturing (13), 1998, pp. 57-76.

Zimmer, I., "A Lens Study of the Prediction of Corporate Failure by Bank Loan Officers," Journal of Accounting Research (18:2), 1980, pp. 629-636. 